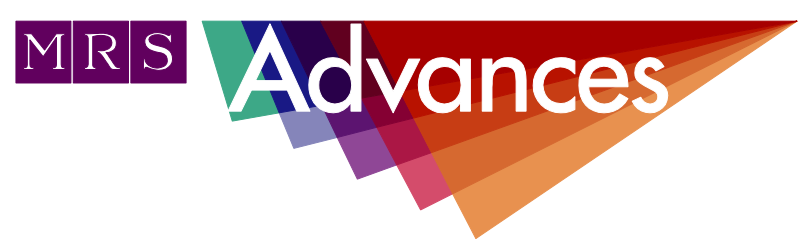

\title{
Delivery of Immunomodulatory Microparticles in a Murine Model of Rotator Cuff Tear
}

\author{
Jack R. Krieger ${ }^{1}$, Mary Caitlin P. Sok ${ }^{1}$, Thomas C. Turner ${ }^{1}$, and Edward A. Botchwey ${ }^{1}$ \\ ${ }^{1}$ Wallace H. Coulter Department of Biomedical Engineering, Georgia Institute of Technology and \\ Emory University, 313 Ferst Drive, Atlanta, Georgia 30332, United States
}

\begin{abstract}
Full thickness rotator cuff tears (RCT) and the associated muscle degeneration that results due to this injury presents a significant clinical burden. The prevention or recovery from this degeneration requires the synchronized behavior of many cells that participate in regeneration. Strategies that tune the inflammatory cascade that is initiated after injury serves as a powerful way to influence tissue repair. Here, we use the local, sustained delivery of the immunomodulatory small molecule FTY720 to examine whether the recruitment of pro-regenerative myeloid cells affects the healing outcome. We find that PLGA microparticles have an atrophic effect on the muscle that is ameliorated with the release of FTY720. However, the inability of FTY720 delivery to induce pro-regenerative monocyte and macrophage recruitment and our findings demonstrating enrichment of $C D 4+T$ cells suggest that effects of this small molecule are context dependent and that the underlying mechanisms behind this RCT associated muscle degeneration require further studies.
\end{abstract}

\section{INTRODUCTION}

Full-thickness rotator cuff tears (RCTs) are a significant clinical burden that are present in more than $20 \%$ of the population [1]. RCTs increase in frequency and severity with age causing significant pain and functional deficiency [2-6]. As a result of RCTs, associated muscles are degenerated through processes that include muscle retraction and atrophy, fatty infiltration, and fibrosis. This associated muscle degeneration is a strong predictor of patient morbidities such as pain, functional deficiency, and post-surgical tear recurrence, and is not reversed by tendon repair $[7,8]$. Thus, prevention or reversal of muscle degeneration due to RCT is a major unmet clinical need. 
Strategies that enhance the early recruitment of non- classical monocytes have been shown to lead to subsequent increases in arteriolar expansion and angiogenic activity $[9,10]$. These recruited monocytes can also differentiate into alternatively activated wound healing ("M2") macrophages that are associated with a number of pro-regenerative activities such as extracellular remodelling, secretion of anti-inflammatory cytokines, and resolution of inflammation [11-14]. FTY720, an agonist of sphingosine-1-phosphate receptor (SIPR) 3, has been shown to recruit non-classical monocytes, stimulate the in situ generation of wound healing macrophages and promote arteriogenesis $[9,15]$. Thus, the delivery of FTY720 has the potential to shift the injury microenvironment from pro-inflammatory to proregenerative and help promote muscle regeneration [16].

PLGA microparticles carriers are a common method used for the sustained release of small molecules. Therefore, using PLGA microparticles, a sustained release of FTY720 over several weeks is achieved in this study to therapeutically immunomodulate the RCT-associated muscle injury environment. In the present study, we demonstrate that while control PLGA microparticles exacerbate atrophy, FTY720 loaded microparticles had no effect on atrophy. However, a daily bolus injection of FTY720 exhibits broad pro-inflammatory effects but is hindered by the additional injury introduced by repeated injections. These results indicate that the immunomodulatory effects of FTY720 could be very context dependent and/or that a more suitable biomaterial carrier for sustained release should be used. Additionally, more studies investigating the mechanisms behind this RCTassociated muscle degeneration are necessary.

\section{MATERIALS AND METHODS}

\section{Mouse model of massive rotator cuff injury}

All animal procedures were conducted according to protocols approved by the Georgia Tech Institutional Animal Care and Use Committee. Male C57bl/6 mice aged 8 weeks were anesthetized using vaporized isoflurane. Sustained-release buprenorphine was administered i.p. for analgesia. The right shoulder served as the injured group and the contralateral shoulder served as the uninjured control group. The right arm and chest were shaved, depilated, and sterilized using triplicate alternating washes of alcohol and chlorhexidine. Using a No. 11 scalpel blade, a lateral incision in the skin was made from the midline to the humeral head. Fascia was removed by blunt dissection. To expose the glenohumeral joint, the deltoid was split by making a $\sim 5 \mathrm{~mm}$ incision from the clavicle to the superior-lateral region of the humerus. The supraspinatus and infraspinatus tendons were sharply transected using the scalpel blade. The suprascapular nerve (SSN) was located by creating a small incision in the pectoralis major and bluntly dissecting the muscle. The SSN was severed using surgical microscissors. The deltoid and pectoralis were closed using absorbable sutures and the skin was closed using wound clips. Mice were allowed unrestricted ambulation after awakening from anesthesia.

\section{Flow cytometry}

For flow cytometric muscle analysis, mice were euthanized by $\mathrm{CO} 2$ asphyxiation 7 days after injury to analyze cellular inflammation in the muscle. Tissue was minced, digested in $1 \mathrm{mg} / \mathrm{mL}$ collagenase IA for $45 \mathrm{~min}$ at $37^{\circ} \mathrm{C}$, filtered through membranes with $40 \mu \mathrm{m}$ pore size, and resuspended in $3 \%$ FBS for 
immunostaining. Cell suspensions were immunostained for $30 \mathrm{~min}$ on ice followed by fixation in 2\% PFA for $10 \mathrm{~min}$ and addition of CountBrightTM Absolute Counting Beads. The following antibody panel was used: MerTK-PE (clone 108928; R\&D Systems), CCR7-PE/Cy7 (clone 4B12; BioLegend), CD3-FITC (17A2; BioLegend), CD25-PerCP/Cy5.5 (clone PC61; BioLegend), Ly6C-APC (clone HK1.4, BioLegend), Ly6G-APC/Cy7 (clone 1A8; BioLegend), CD11c-BV421 (clone N418; BioLegend), CD11b-BV510 (clone M1/70; BioLegend), CD206-BV605 (clone C068C2; BioLegend), CD64-BV711 (clone X54-5/7.1; BioLegend), and CD4-BV785 (clone GK1.5; BioLegend). Samples were run on a BD FACS Aria IIIu cytometer and data was analyzed using FlowJo software. Cells were immunophenotyped according to the following gating scheme: macrophage, MerTK+CD64+; dendritic cell, NOT(MerTK+CD64+)CD11c+; monocyte, NOT(MerTK+CD64+)CD11c-CD11b+SSClo; neutrophil, Ly6G+SSChi; T lymphocyte, CD3+; helper T lymphocyte, CD3+CD4+; regulatory $\mathrm{T}$ lymphocyte (Treg), CD3+CD4+CD25+.

\section{FTY720-loaded PLGA microparticle fabrication}

FTY720-loaded PLGA microparticles were fabricated using established single emulsion technique. Briefly, FTY720 (Cayman Chemical) dissolved in DMSO $(20 \mathrm{mg} / \mathrm{mL}$ ) was mixed at a mass ratio of 1:70 ( 9 week study) or 1:150 (7 day study) with PLGA (50:50 copolymer ratio) dissolved in methylene chloride $(20 \%$ $\mathrm{wt} / \mathrm{v}$ ). Polymer solution was added dropwise to poly(vinyl alchohol) solution (2\% $\mathrm{w} / \mathrm{v}$, aqueous) under constant homogenization at 4000 RPM for 1 min. Resultant microparticle suspension was stirred for $3 \mathrm{~h}$ to evaporate solvent, sieved through a porous membrane ( $40 \mu \mathrm{m}$ pore size) to isolate particles larger than $40 \mu \mathrm{m}$, and washed 3 times in deionized water. Control PLGA microparticles were fabricated using the same process but with FTY720 absent from the initial DMSO solution. For in vivo studies, equivalent fractions of the total batch volume were taken from FTY720 and PLGA microparticle batches.

\section{$\underline{\text { In vitro release of FTY720 from PLGA microparticles }}$}

FTY720-encapsulating PLGA microparticles were suspended in simulated body fluid with $4 \%$ fatty acid-free BSA and incubated at $37^{\circ} \mathrm{C}$. Media was exchanged at designated time points to quantify FTY720. FTY720 was isolated from samples by lipid extraction. Briefly, samples were added to $2: 1 \mathrm{CH}_{3} \mathrm{OH}: \mathrm{CHCl}_{3}$ solution and heated at $48^{\circ} \mathrm{C}$ overnight. Glycerophospholipids were degraded by heating at $37 \mathrm{C}$ for $2 \mathrm{~h}$ in the presence of $\mathrm{KOH}$. The $\mathrm{pH}$ was neutralized with acetic acid, solvent was evaporated using a SpeedVac concentrator, and samples were dissolved in 72:29:1 $\mathrm{CH}_{3} \mathrm{OH}: \mathrm{H}_{2} \mathrm{O}: \mathrm{HCOOH}$ solution. For LC-MS/MS analysis, samples were injected into a C18 chromatography column upstream of a Micromass Quattro LC triple quadrupole tandem mass spectrometer operating under electrospray ionization.

\section{Intramuscular injection of therapeutics}

Microparticles (20 $\mu \mathrm{L}$ volume) were injected directly into the SS muscle during surgery using insulin syringe. To administer soluble FTY720 daily, FTY720 was first dissolved in DMSO at a concentration of $20 \mathrm{mg} / \mathrm{mL}$ and further diluted in sterile saline. Mice were anesthetized with vaporized isoflurane, the scapula was gently restrained, the insulin syringe was passed through the skin into the SS muscle, and FTY720 solution (20 $\mu \mathrm{L}$ sterile saline) was injected. 


\section{$\underline{\text { Statistical analysis }}$}

Statistical comparisons were conducted in GraphPad Prism using repeated measures two-way ANOVA followed by Sidak multiple comparisons test or ordinary one-way ANOVA followed by Holm-Sidak post-hoc test.

\section{RESULTS}

\section{Intramuscular injection of bolus FTY720 stimulates pro-inflammatory immune cell infiltration}

We first investigated the effect of on-site delivery of FTY720 in the absence of biomaterial carriers to elucidate the immune response to the drug alone. Bolus FTY720 (1 $\mu \mathrm{g}$ in $20 \mu \mathrm{L})$ was administered daily via intramuscular injection starting at the time of surgery, and immune cell infiltration was assessed at day 7 via flow cytometry.
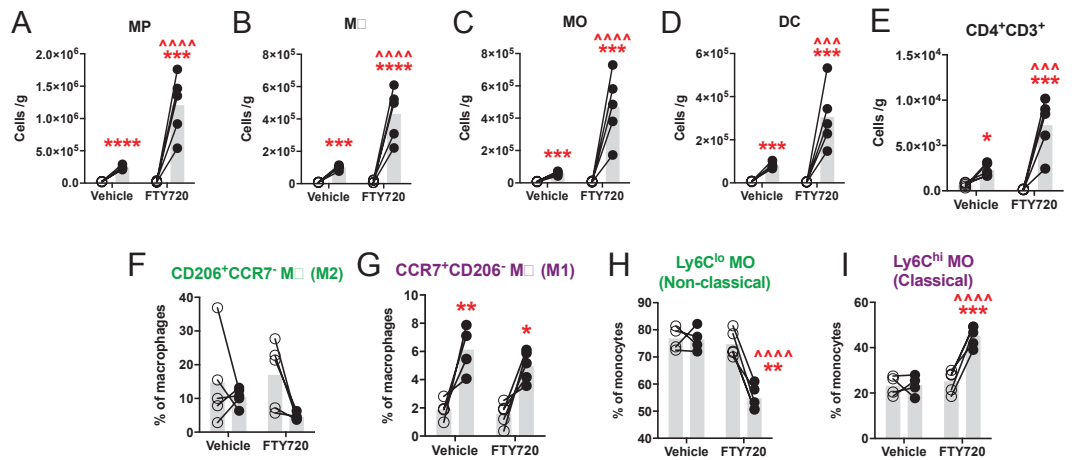

Figure 1. Daily on-site delivery of bolus FTY720 affects immune cell infiltration. Flow cytometric analysis of mononuclear phagocytes (A), macrophages (B), monocytes (C), dendritic cells (D), and T-helper cells (E) in SS muscle at day 7 compared to vehicle control and uninjured contralateral control. Analysis of mononuclear phagocyte populations M2-like macrophages (F), M1-like macrophages (G), Ly6C-lo non-classical monocytes (H), and Ly6C-hi classical monocyte (I) frequencies within their respective parent populations at day 7. Open circles indicate uninjured contralateral control, filled circles correspond to injury. ${ }^{*} P<0.05,{ }^{* *} P<0.01,{ }^{* * *} P<0.001$, ${ }^{* * * *} P<0.0001$ compared to uninjured contralateral control, ${ }^{\wedge \wedge \wedge} P<0.001,{ }^{\wedge} \wedge \wedge P<<0.0001$ compared to vehicle control, as determined by two-way repeated measures ANOVA with post-hoc multiple comparisons test $(n=5)$.

Daily FTY720 caused dramatically increased accumulation of mononuclear phagocytes, macrophages, monocytes, dendritic cells, and CD4+ T-helper cells compared to vehicle control (Figure 1 a-e). FTY720 did not affect the proportion of M2-like macrophages compared to vehicle (Figure 1f); however, both vehicle and FTY720 increased the proportion of CCR7 ${ }^{+}$CD206- M1-like macrophages compared to uninjured contralateral controls (Figure 1g). FTY720 decreased the proportion of Ly6C lo non-classical monocytes (Figure 1h) and increased the proportion of 
Ly6Chi classical monocytes (Figure 1i). Daily FTY720 did not affect blood levels of circulating monocytes (Figure 2a,b), but did cause lymphopenia with regard to both the $\mathrm{CD}_{4}{ }^{+} \mathrm{T}$-helper and $\mathrm{CD}^{+}$cytotoxic $\mathrm{T}$ lymphocyte subpopulations of circulating T lymphocytes (Figure 2c,d).
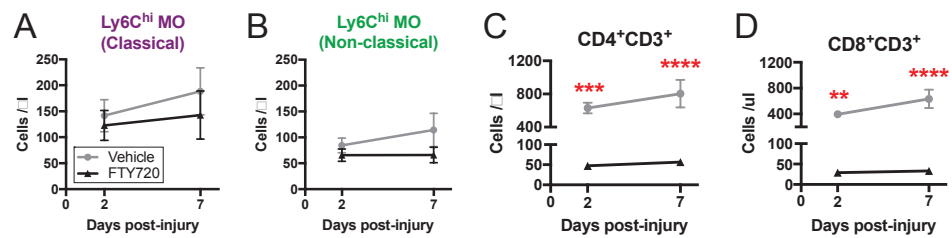

Figure 2. Immune cell count in blood circulation in response to RCT and daily on-site delivery of FTY720. (A) Ly6C-hi classical monocytes, (B) Ly6C-lo non-classical monocytes, (C) CD4+ T-helper lymphocytes, and (D) CD8+ cytotoxic T lymphocytes. ${ }^{* *} P<0.01,{ }^{* * *} P<0.001,{ }^{* * * *} P<0.0001$ compared to vehicle control as determined by two-way repeated measures ANOVA with post-hoc multiple comparisons test $(n=5)$.

\section{Local, sustained delivery of FTY720 via PLGA microparticles}

Because FTY720 affects both the recruitment and cytokine elaboration of MO [9], and because sustained release of FTY720 over several weeks can be achieved using PLGA microparticle carriers [17], we next attempted therapeutic immunomodulation using local delivery of FTY720-loaded PLGA microparticles (Figure 3a). To achieve sustained local delivery of FTY720 during the progression of immune cell infiltration, 50:50 PLGA microparticles encapsulating FTY720 (total dose encapsulated in microparticles $=10 \mu \mathrm{g}$, drug:polymer ratio $=1: 150$ ) were injected into supraspinatus muscle at the time of surgery. PLGA microparticles release FTY720 in vitro with an initial burst followed by relatively linear release (Figure 3b). Delivery of microparticles loaded with $10 \mu \mathrm{g}$ of FTY720 does not affect absolute mononuclear phagocyte count, T lymphocyte count, or TH/Treg count at day 7; however, the frequency of regulatory $\mathrm{T}$ cells in the T-helper and $\mathrm{T}$ lymphocyte populations is decreased in the FTY720 microparticle group compared to unloaded PLGA microparticles and vehicle control (Figure 3c-e). 


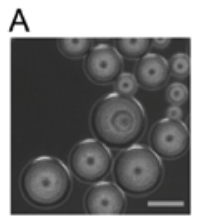

B

C $\mathrm{CD}^{2} 5^{+\mathrm{CD}^{+} \mathrm{CD}^{+}}$
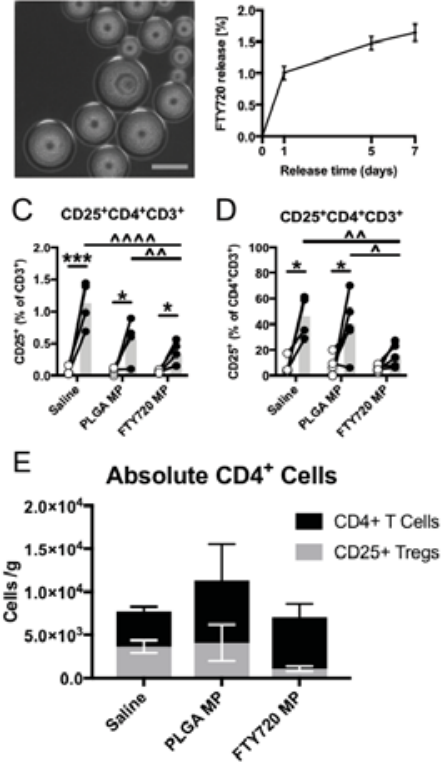

Figure 3. Effect of FTY720-releasing PLGA microparticles at acute day 7 time point. (A) Light microscopy image of PLGA microparticles (scale bar $=100 \mu \mathrm{m}$ ). (B) FTY720 release kinetics in vitro from PLGA (1:150) microparticles. FTY720 was released into simulated body fluid with $4 \%$ fatty acid-free BSA and incubated at $37^{\circ} \mathrm{C}$. Total dose delivered in vivo was $10 \mu \mathrm{g}$. There was an observed increase in Treg cells across all groups after injury, but FTY720 release from microparticles results in a lower frequency of Treg cells within the T lymphocyte (C) and helper T lymphocyte (D) populations in SS muscle at day 7 compared to vehicle and unloaded MP controls ( $n=4-5$, two-way ANOVA with repeated measures). Open circles indicate uninjured contralateral control, filled circles correspond to injury. (E) Absolute quantification of CD4+ T cells showing the CD25+ Treg population. There were no differences across all groups in the absolute numbers of CD4+ or CD25+ T Cells. ${ }^{*} P<0.05$, *** $P<0.001, \wedge P<0.05, \wedge \wedge P<0.01, \wedge \wedge \wedge \wedge P<0.0001$.

We then fabricated PLGA microparticles with a drug:polymer ratio of 1:70 to attain sustained release of FTY720 over the course of five weeks (Figure 4a). Microparticle-mediated delivery of $1.1 \mu \mathrm{g}$ FTY720 does not significantly affect supraspinatus wet mass at week 9 compared to untreated control and PLGA MPs; however, unloaded PLGA MPs significantly decrease mass compared to untreated control (Figure 4b). Similarly, unloaded PLGA MPs increase accumulation of CD68+ $\mathrm{M} \Phi$ and $\mathrm{CD}^{206}{ }^{+} \mathrm{CD} 8^{+} \mathrm{M} 2$-like $\mathrm{M} \Phi$ at week 9 compared to untreated control, whereas FTY720 release returns $\mathrm{CD}^{2} 8^{+}$and $\mathrm{CD} 206^{+} \mathrm{CD} 68^{+}$counts to the same level as untreated control (Figure 4c-f). However, decrease in muscle mass did not correlate strongly with $\mathrm{CD} 206^{+} \mathrm{CD} 68^{+}$count $\left(\mathrm{r}^{2}=0.18\right)$, (Figure $\mathbf{4 g}$ ), and the slope of the regression line did not significantly differ from zero, indicating that the mechanism of PLGA MP muscle degeneration is independent of the action of macrophages. 

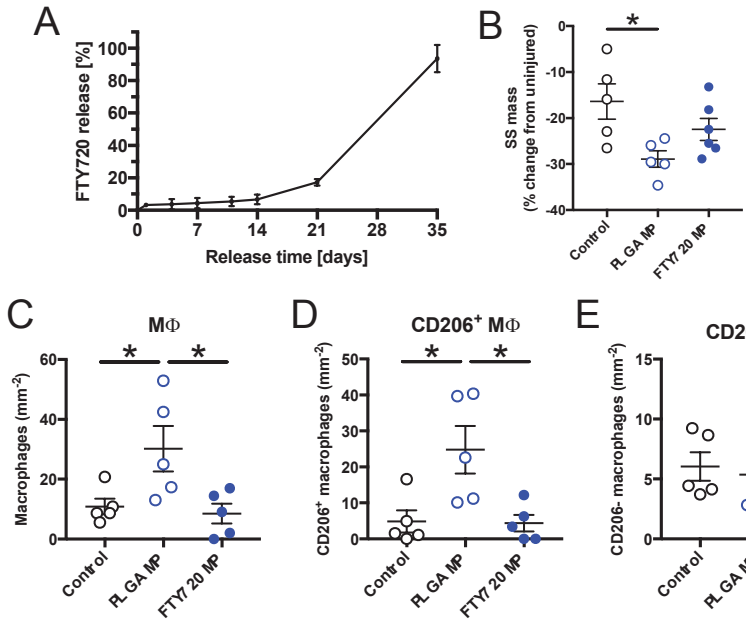

$E$
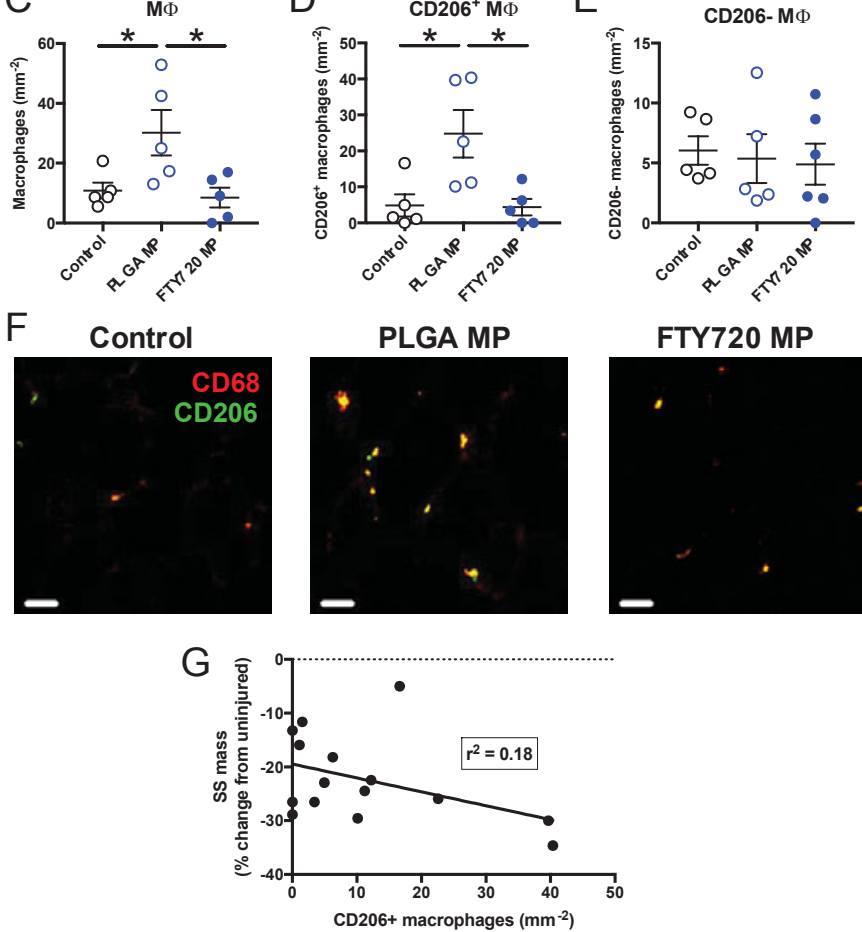

Figure 4. Effect of FTY720-releasing PLGA microparticles at chronic week 9 time point. (A) FTY720 release kinetics in vitro from PLGA (1:70) microparticles. (B) PLGA microparticles decrease the percent change in wet mass between injured SS and uninjured contralateral SS (B). Macrophage (C), CD206+ macrophage (D), and CD206- macrophage (E) counts as determined by cross-sectional IHC (F; scale bar = 20 $\mu \mathrm{m})$. Change in SS mass does not negatively correlate with CD206+ macrophages $\left(\mathrm{G} ; \mathrm{r}^{2}=0.18\right) .{ }^{*} P<0.05$ by ANOVA ( $\mathrm{n}=5-6$ animals). 


\section{DISCUSSION}

To investigate on-site FTY720 in the absence of contributions to the immune response by PLGA microparticles, bolus FTY720 was injected into supraspinatus muscle daily. FTY720 exhibited broad pro-inflammatory effects, increasing the accumulation of all mononuclear phagocyte populations, increasing the frequency of pro-inflammatory classical monocytes and M1-like macrophages, and decreasing the frequency of non-classical monocytes. FTY720-induced patterns of mononuclear phagocyte accumulation were unlikely to result from systemic effects on circulating monocytes, given that blood counts of classical and nonclassical monocytes did not differ between FTY720 and control. FTY720 dramatically increased helper $\mathrm{T}$ cell accumulation despite causing lymphopenia in blood. These immunomodulatory effects due to daily injection of FTY720 may be explained by additional injury stimuli introduced by the injections. Repeated injections likely injure myofibers, thereby creating a profile of DAMPs, cytokines, and endothelial adhesion signatures that differ from supraspinatus muscle experiencing indirect injury via tendon and nerve transection.

In order to mitigate the stimulation of the immune response due to repeated traumatic muscle injury, subsequent studies of therapeutic drug delivery pivoted to local delivery of FTY720-encapsulating PLGA microparticles for multiple reasons. First, our lab previously showed that biomaterial FTY720 delivery can stimulate MO in vitro and injured skin in vivo causing cytokine secretion to shift the balance from pro-inflammatory to pro-regenerative factors. Secondly, PLGA microparticles can sustain the release of FTY720 over multiple weeks, whereas attempts to utilize other biomaterials in microparticle fabrications exhibit rapid release of FTY720 by $24 \mathrm{~h}$, decreasing their utility in injury models that have sustained inflammatory reactions.

At the early seven-day time point, FTY720 release from PLGA microparticles decreases the percentage of Treg cells within the helper $\mathrm{T}$ cell and total T cell populations, without an observed decrease in absolute Treg number. Decreased Treg frequency may be caused by multiple processes, including decreased recruitment, proliferation, survival, retention, or differentiation of Treg, or an increase in these processes in other T lymphocyte populations. However, both Tregs and T helper type 2 cells have both been implicated in the process of muscle repair and regeneration [18,19]. Our observed decrease in the overall frequency of CD25+ Tregs within the T helper cell population warrants more exploration to fully quantify the phenotype of these CD4+ T cells generated by the delivery of FTY720 MPs. At the late 9-week time point, FTY720 release had no significant effect on supraspinatus atrophy, whereas control PLGA microparticles exacerbated atrophy. PLGA microparticles increased accumulation of macrophages, particularly CD206+ M2-like macrophages. Supraspinatus atrophy did not correlate with CD206+ macrophage count, suggesting that CD206+ macrophages likely do not exert atrophic effects on the supraspinatus muscle. The acidic products of PLGA degradation, lactic acid and glycolic acid, may negatively regulate muscle mass [20], but additional experiments would be necessary to test this hypothesis.

Inability of local delivery of FTY720 to induce pro-regenerative monocyte and macrophage recruitment and protein production after murine RCT contrasts with prior studies indicating such effects in the murine dorsal skin window model $[9,10,21]$. The disparity may suggest that the effects of FTY720 on inflammation are highly context-dependent. Given our findings demonstrating the local enrichment of CD4+ $\mathrm{T}$ cell populations, the therapeutic window for immune modulation in complex RCT injuries may be dependent on lymphoid, and not myeloid, immune 
cells. To identify additional therapeutic targets, deeper investigation of the mechanisms underlying supraspinatus degeneration and regeneration are necessary.

\section{ACKNOWLEDGMENTS}

We thank the core facilities staff of the Parker H. Petit Institute for Bioengineering and Bioscience for technical expertise and assistance (particularly Andrew Shaw and Nadia Boguslavsky), and the Physiological Research Lab staff for guidance on in vivo studies. This work was supported by the National Institutes of Health grants R01AR056445-01A2 and R01DE019935-0.

\section{References}

[1] H. Minagawa, N. Yamamoto, H. Abe, M. Fukuda, N. Seki, K. Kikuchi, H. Kijima, E. Itoi, J Orthop 10 (1), 8-12 (2013).

[2] J. Ozaki, S. Fujimoto, Y. Nakagawa, K. Masuhara, S. Tamai, J Bone Joint Surg Am 70 (8), 1224-30 (1988).

[3] K. Yamaguchi, K. Ditsios, W.D. Middleton, C.F. Hildebolt, L.M. Galatz, S.A. Teefey, J Bone Joint Surg Am 88 (8), 1699-704 (2006).

[4] S.N. Sambandam, V. Khanna, A. Gul, V. Mounasamy, World J Orthop 6 (11), $902-18$ (2015).

[5] J. Hsu, J.D. Keener, Oper Tech Orthop 25 (1), 2-9 (2015).

6] S. Tempelhof, S. Rupp, R. Seil, J Shoulder Elbow Surg 8 (4), 296-9 (1999).

[7] J.N. Gladstone, J.Y. Bishop, I.K. Lo, E.L. Flatow, Am J Sports Med 35 (5), $719-28$ (2007)

8] C. Gerber, A.G. Schneeberger, H. Hoppeler, D.C. Meyer, J Shoulder Elbow Surg 16 (6), 691-6 (2007).

[9] A.O. Awojoodu, M.E. Ogle, L.S. Sefcik, D.T. Bowers, K. Martin, K.L. Brayman, K.R. Lynch, S.M. Peirce-Cottler, E. Botchwey, Proc Natl Acad Sci U S A 110 (34), 13785-90 (2013).

[10] J.R. Krieger, M.E. Ogle, J. McFaline-Figueroa, C.E. Segar, J.S. Temenoff, E.A. Botchwey, Biomaterials 77, 280-90 (2016).

[11] S.J. Jenkins, D. Ruckerl, P.C. Cook, L.H. Jones, F.D. Finkelman, N. van Rooijen, A.S. MacDonald, J.E. Allen, Science 332 (6035), 1284-8 (2011).

[12] D.M. Mosser, J.P. Edwards, Nat Rev Immunol 8 (12), 958-69 (2008).

[13] D. Madsen, D. Leonard, A. Masedunskas, A. Moyer, H. Jürgensen, D. Peters, P. Amornphimoltham, A. Selvaraj, S. Yamada, D. Brenner, S. Burgdorf, L. Engelholm, N. Behrendt, K. Holmbeck, R. Weigert and T. Bugge, The Journal Of Cell Biology 202, 951-966 (2013).

[14] K.A. Kigerl, J.C. Gensel, D.P. Ankeny, J.K. Alexander, D.J. Donnelly, P.G. Popovich, J Neurosci 29(43), 13435-44 (2009).

[15] C.E. Olingy, C.L. San Emeterio, M.E. Ogle, J.R. Krieger, A.C. Bruce, D.D. Pfau, B.T. Jordan, S.M. Peirce, E.A. Botchwey, Sci Rep 7(1), 447 (2017).

[16] C.L. San Emeterio, C.E. Olingy, Y. Chu, E.A. Botchwey, Biomaterials 117, 32-43 (2017).

[17] A. Das, D.A. Barker, T. Wang, C.M. Lau, Y. Lin, E.A. Botchwey, PLoS One 9(7), e101276 (2014).

[18] D. Burzyn, W. Kuswanto, D. Kolodin, J. Shadrach, M. Cerletti, Y. Jang, E. Sefik, T. Tan, A. Wagers, C. Benoist and D. Mathis, Cell 155(6), 1282-95 (2013).

[19] K. Sadtler, K. Estrellas, B. Allen, M. Wolf, H. Fan, A. Tam, C. Patel, B. Luber, H. Wang, K. Wagner, J. Powell, F. Housseau, D. Pardoll and J. Elisseeff, Science 352, (2016).

[20] X.H. Wang, W.E. Mitch, Nat Rev Nephrol 10 (9), 504-16 (2014).

[21] M.E. Ogle, J.R. Krieger, L.E. Tellier, J. McFaline-Figueroa, J.S. Temenoff, E.A. Botchwey, ACS Biomaterials Science \& Engineering (2017). 